\title{
INHALT DES JAHRGANGS 1956
}

\section{Aufsätze}

Heft-Nr. Seite

Berkov, P. N., Aus der Geschichte der deutsch-russischen Theaterbeziehungen im XVIII. Jahrhundert (übersetzt von H. Raab) . . . . . . 49

Bielfeldt, H. H., Fragen des russisch-deutschen Wörterbuches . . . . . 219

DudeK, G., Die Kompositionsformen in der frühen Lyrik N. A. Nekrassows . . $\quad 3 \quad 81$

Düwel, W. u. Bathe, I., Cennyševskijs Rezension der russischen SchillerAusgabe von N. V. Gerbel' . . . . . . . . . . . . . . . . . . . . .

EICHLER, E., Eine westslawische Bezeichnung für ,Sumpf, Feuchtigkeit": altsorbisch *mroka. . . . . . . . . . . . . . . . . . . . . . . . . . 339

FahlBUSCH, L., Slawisches aus Daubes Wörterbuch der Altenburger Mundart. $1 \quad 28$

Falkenhahn, V., Entstehung, Entwicklung und Ende der urslawischen Sprachgemeinschaft in polnischen Veröffentlichungen von T. Lehr-Spławiński.

-, Zur Frage nach den treibenden Kräften bei der Gestaltung der polnischen Verbalrektion . . . . . . . . . . . . . . . .

FeYL, O., J. F. Fries, F. G. Hand und Rußland . . . . . . . . . . . . .

-, Zum 100. Todestag des tschechischen Publizisten und Dichters Karel Havliček-Borovský .

Fischer, R., Erlebnisse August Schleichers in der Bach'schen Ära . . . . .

-, Ferdinand Liewehr zum 60. Geburtstag . . . . . . . . . . . . . . . 4

GaBKA, K., Über die sogenannten ,weiterführenden Nebensätze" im Russi-

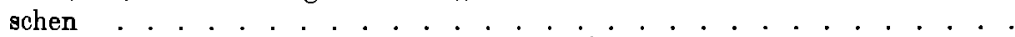

Hofman, A., Maximilian Heine, ein Beitrag zur deutsch-slawischen Wechselseitigkeit . . . . . . . . . . . . . . . . . . . . . . . . . . .

HYRŠLOVA, K., Friedrich Schiller und die tschechische nationale Wiedergeburt (übersetzt von A. Eckelt) . . . . . . . . . . . . . . . . . . . . . .

JAROSCH, G., Edmund Schneeweis zum 70. Geburtstag . . . . . . . . . .

KNOBLOCH, J., Zur Herkunft und Bedeutungsgeschichte von tschech. švanda.

KOMÁREK, M., Zur Entwicklung des tschechischen Vokalsystems . . . . . .

Kujew, K. M., Wie Adam Mickiewicz und Konrad Rylejew miteinander bekannt wurden . . . . . . . . . . . . . . . . . . . . . . .

LiEWEHR, F., Einiges über slawische Flexionsendungen . . . . . . . . . .

—, Serbokroatisch und bulgarisch nemoj und Verwandtes . . . . . . . . . .

—, Utber expressive Sprachmittel im Slawischen . . . . . . . . . . . . . . 1

-, Zum Vers der Kiewer Blätter . . . . . . . . . . . . . . . . . . . . 2

MACHEK, V., Slavisch ot-, baltisch at-. . . . . . . . . . . . . . . . . . 1

-, Expressive Vokaldehnung in einigen slavischen Nomina . . . . . . . . 4

Peukert, H., Die nichtkatholischen Slawen und der Josefinismus . . . . . 4

RAAB, H., Anfänge einer russischen Literaturbetrachtung in deutschen Zeitschriften . . . . . . . . . . . . . . . . . . . . . . . . . . . . . 4108

—, Die Lyrik Schillers in früher russischer Utbersetzung . . . . . . . . . . . . . 140

REPP, F., Zu den Wiener Bruchstücken der Alexandreis. . . . . . . . . . . . . 132

-, Textkritische Untersuchungen zur UUberlieferung des Vévoda Arnošt im Codex Baworowský. . . . . . . . . . . . . . . . . . . . . . . . . . 444

RUNGE, K., Landschafts- und Naturbilder in den Gedichten A. K. Tolstojs . . 4115

SchröPfer, J., Zur inneren Sprachform der Balkanvölker . . . . . . . . . . 44139

Schultz, J., Zur Frequenz polnischer Studenten an der Universität Jena . . 1108 
- Schütz, J., Bulgarisch grъzdav, ,holperig, rauh; heiser" . . . . . . . . . 3

- Stanislav, J., Zur Frage der Slowazismen in den alttschechischen Denkmälern (übersetzt von K. Gabka). . . . . . . . . . . . . . . . . . . 4

, -, Über die Stellung des Slowakischen innerhalb der slawischen Sprachen (übersetzt von E. Eichler) . . . . . . . . . . . . . . . . . . . . . . 2

Tetzner, J., Die Leipziger Neuen Zeitungen von gelehrten Sachen über die Anfänge der Petersburger Akademie . . . . . . . . . . . . . . . . .

Winter, E., J. Dobrovksy und das Domstift von St. Peter in Bautzen . . . -, ,Einige Nachricht von Herrn Simeon Todorski“. Ein Denkmal der deutsch-slawischen Freundschaft im 18. Jahrhundert . . . . . . . . . .

- Zolnai, B., Die geistige Bedeutung des Generalseminars von Preßburg (Bratislava) für Ungarn und die slawischen Völker . . . . . . . . . . .

\section{Materialien}

- FEyL, O., Unbekannte deutsche Briefe und andere Dokumente von Jan Kollár aus Weimar . . . . . . . . . . . . . . . . . . . . . . . . 2121

Ein Brief A. Mickiewicz', angezeigt von O. Fcyl _ . . . . . . . . . . . . . . . . . . . . 3156

REPP, F., Ein alttschechischer Schreibervers in der Hs. Nr. 4831 der Wiener Nationalbibliothek . . . . . . . . . . . . . . . . . . . 1

\section{Literaturberichte, Besprechungen und Anzeigen}

- FEyL, O., Zehn Jahre Slawistik und Osteuropa-Kunde im Spiegel der deutschsprachigen Veröffentlichungen Ostdeutschlands (1945-1955) Teil 1 . . . .

EICHLER, E., Slawistische und germanoslawistische Arbeiten zur Namenforschung aus der DDR. Úbersicht bis März 1955 . . . . . . . . . . . . . . . 1138

FEy L, O., Arbeiten zur Geschichte der deutsch-slawischen Beziehungen . . 137

MatL, J., Das wissenschaftliche Werk Josef Matls seit 1950, besprochen von E. Winter

Kurze Anzeigen von E. Winter . . . . . . . . . . . . . . . . . . . .

Kurze Anzeigen von E. Winter . . . . . . . . . . . . . . . . . . . .

Schuster-Sewc, H., Sorabica . . . . . . . . . . . . . . . . . . . .

Veröffentlichungen zur polnischen Geschichte von F. H. Gentzen . . . . . .

Zeitschriftenartikel über Fragen deutsch-slawischer Wechselseitigkeit von E. Wolfgramm und H. Mohrmann . . . . . . . . . . . . . . . . . .

Dissertationen auf den Gebieten der Slawistik und Osteuropäischen Geschichte, angenommen an den Universitäten der DDR in den Jahren 1945-1955 . .

BAcH, A., Deutsche Namenkunde, besprochen von R. Fischer . . . . . . .

BAHLow, H., Schlesisches Namenbuch, besprochen von R. Fischer . . . .

BĚLIC, J., Dolská nářeči na Moravě, besprochen in ,Eine bedeutsame dialektologische Arbeit" von F. Kopečný . . . . . . . . . . . . . . . . . .

Benz, E. [Hrgb.] Russische Heiligenlegenden, besprochen von F. v. Lilienfeld

Bon Čv, V. I. Израсло дърео високо, besprochen von Ch. Vakarelski . . .

BRÜskE, W., Untersuchungen zur Geschichte des Lutizenbundes, besprochen von E. Donnert. . . . . . . . . . . . . . . . . . . . . . . . . . .

Feyl, O., Exkurse zur Geschichte der südosteuropäischen Beziehungen der Universität Jena, angezeigt von E. Winter . . . . . . . . . . . . . .

Fischer, R., Tschechische Grammatik, besprochen von E. Müller . . . . .

-, Tschechisches Lesebuch, besprochen von H. Siebenschein . . . . . . . . . 
Fischer, R., Probleme der Namenforschung an Orts- und Flurnamen im westlichen Bohmen und in seiner Nachbarschaft, besprochen von E. Müller

Georgi pop Ivaxov, Народни песни и приказки от Софийско и Ботевградско, besprochen ron $\mathrm{Ch}$. Vakarelski

Hofman, A., Friedrich Schiller, besprochen von E. Schneeweis. . . . . . .

KorespondenciJa Vatroslava JAGiĆA, besprochen von H. Röbel . . . . .

Krovika Thietmara, besprochen von E. Donnert . . . . * . . • • • . .

IJIEWEHR, F., Slawische Sprachwissenschaft in Einzeldarstellungen, besprochen von J. Knobloch. . . . . . . . . . . . . . . . . . . . .

MaChEK, V., Ceská a slovenská jména rostlin, besprochen von R. Fischer . .

Mijatev, K., Българският сукман, besprochen von Ch. Vakarelski . . . .

Nowák-Neumaxx, M. und Nedo, P., Die Tracht der Sorben um Schleife, besprochen von $0 . v$ Zaborsky . . . . . . . . . . . . . . . . . . .

ORMIS, J. V., Bibliografia Jana Kollára, besprochen von O. Feyl . . . . . .

ОчЕРКИ ИСТоРји СССР. Период феодализма IX-XV в8., beøprochen von E. Donnert . . . . . . . . . . . . . . . . . . . . . . . . . . . .

Parchomenko, M., Ivan Franko i russkaja literatura, besprochen von

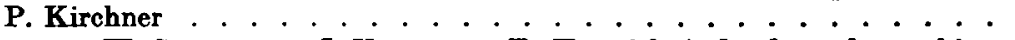

Philipp, W.-Smolitsch, I.-Valjavec, F., Verzeichnis des deutschaprachigen Schrifttums 1939-1952 zur Geschichte Ostexiropes und Südosteuropas, besprochen von P. Hoffmann . . . . . . . . . . . . . . . . . .

Preidel, H., Die Anfänge der slawischen Besiedlung Böhmons und Mährens, besprochen von R. Fischer . . . . . . . . . . . . . . . . . . . .

v. RAUch, G., Die neuere Geschichte $1500-1815$ in der sowjetiechen Geschichtsschreibung der Gegenwart, angezeigt von E. Winter and P. Hoffmann

Rosenkranz, B., Historische Lant- und Formenlehre des Altbalgarisohen, besprochen von H. H. Bielfeldt . . . . . . . . . . . . . . . . . . .

SetschkarefF, V., N. V. Gogol: Leben und Schaffen, besprochen von F. v. Lilienfeld . . . . . . . . . . . . . . . . . . . . . . . . .

Sieber, F., Bunte Möbel der Oberlansitz, besprochen von O. v. Zaboraky . . Siegmann, F., Die Musik im Leben und Schaffen der russischen Romantiker, besprochen von L. Fahlbusch . . . . . . . . . . . . . . . . .

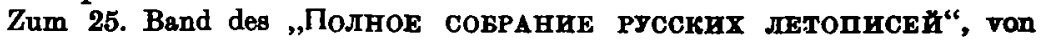
K. Gabka . . . . . . . . . . . . . . . . . . . . . . . . . . . . .

Stanislav, J., Slovensky juh $v$ stredoveku, bésprochen von R. Fischer. . . STöckr, G., Rusøisohes Mittolalter and cowjetisohe Mediaevistik, angeneigt von E. Winter . . . . . . . . . . . . . . . . . . . . . . . . . . .

SIMEK, E., Chebsko - dnešní nejzépadnejłł slovanské územi - v staré dobð, besprochen von R. Fischer . . . . . . . . . . . . . . . . . . . . .

TASzYCKI, W., Slowiańskie nazwy miejscowe, besprochen von E. Fichler . .

PRAŽska UnIVERsita Moskevske UNIVERsité, besprochen von 0 . Feyl . .

Veleva, M. [Hrgb.] Български народни носии и шевици, besprochen von Ch. Vakarelski

WINTER, E,. Die tschechische und slowakische Emigration in Deutachland im 17. und 18. Jahrhundert, besprochen von H. Rösel . . . . . . . . . . .

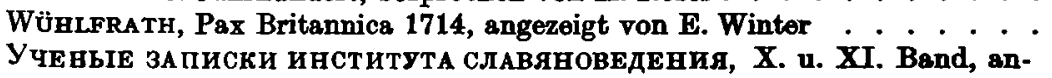
gezeigt von $\mathrm{E}$. Winter . . . . . . . . . . . . . . . . . . . 